\title{
Costs and Efficiency of Integrating Family Planning into Antiretroviral Therapy Services in Zambia: A Mixed Method Comparison of the Fully Integrated Versus Referral Models
}

\section{Sophie Faye*, Benjamin Johns and Elaine Baruwa}

Abt Associates Inc., Montgomery Avenue Suite, Bethesda, MD, USA

\begin{abstract}
Background: Integrating HIV and family planning (FP) services can improve health outcomes, continuity of care, and efficiency. However studies on its cost-efficiency are lacking. The objective of this study is to assess and compare the cost-efficiency of two models of FP/HIV integration in Zambia.
\end{abstract}

Methods: We considered two integration models - "internal referral" (IR), where patients receive FP counselling within the HIV clinic and a referral to the FP clinic in the same facility for FP method, and "one-stopshop" (OSS), where patients receive FP counselling and a FP method within the HIV clinic. We compared the efficiency of the models using the unit cost per antiretroviral therapy (ART) patient provided with FP services. We also computed the percentage of missed opportunities for FP services provision; when patients with identified FP needs are not offered services.

Results: The unit costs per patient provided with FP method was USD \$261 on average for the OSS model and USD $\$ 267$ on average for the IR model. We found no statistically significant difference in unit costs between the two models $(P=0.36)$. On average, the OSS model had 25 percent missed opportunities for FP counselling compared to 33 percent for the IR model but the difference was also not statistically significant $(P=0.3)$.

Conclusion: A fully integrated model of FP/ART services (OSS) is not necessarily more cost-efficient than the referral model as the performance of each depends on the service delivery settings.

Keywords: Integration; Family planning; Antiretroviral therapy; Cost-efficiency

\section{Introduction}

The potential for providing continuity of care to those living with HIV has made service integration a priority in the fight for an AIDS-free generation among donors, governments, and implementers [1]. Studies have found that unmet need for FP is greater in HIV positive women than among their HIV negative counterparts [2]. In that context, integrating FP and HIV services can prevent more unintended pregnancies for HIV positive women. Furthermore, prevention of unintended pregnancies among HIV positive women reduces the number of mother-to-child HIV transmissions [3]. It also decreases overall maternal mortality as HIV positive women have eight times higher risk of a pregnancy-related death [4]. Providing FP in HIV service settings also has qualitative benefits: a study in Kenya found that HIV positive women preferred to receive FP services from HIV providers as compared to other providers due to better confidentiality [5].

Despite the recognized clinical benefits of $\mathrm{FP} / \mathrm{HIV}$ integration, evidence documenting its cost-efficiency is limited [6-9]. The recent Integra Initiative, funded by the Bill \& Melinda Gates Foundation, partly addressed this evidence gap but it's costing studies considered integration of HIV services into various sexual and reproductive health (SRH) services, including Family Planning [10]. In contrast to the Integra Initiative, the focus of this study is on the integration of FP services into HIV services. A recent literature review focusing on costs and efficiency of integrating HIV and other health services found that only 4 out of 46 studies on FP/HIV integration included potential cost savings estimations [11]. The authors noted that little is known about the most cost-efficient way of integrating FP and HIV services.

Since that review, a cluster randomized control trial (RCT) in Kenya compared two models of integrating FP into HIV care and treatment [12]. In that study, health facilities were randomly selected to either implement the fully integrated model (offer FP methods in the HIV clinic (intervention arm)) or implement the internal referral model (refer patients in need of FP method to the FP clinic inside that same facility (control arm)). The results showed an increase of 77 percent in FP method uptake in the intervention arm compared to the control arm. This led to a lower unit cost per service: USD $\$ 70.93$ per woman using the integrated FP method, compared to USD $\$ 80.62$ for the control group.

Following the Kenyan study, the main objective of this study is to estimate and compare cost-efficiency of two FP/ART integration models in Zambia. The two FP/HIV integration models studied are the fully integrated model (OSS) where FP counselling and method are offered from the HIV clinic, and the internal referral (IR) model where an ART patient is referred to the FP clinic in the same health facility for services. We also estimate the percentage of missed opportunities for providing FP services and we describe health provider's perceived implementation challenges and enablers to the integration process.

*Corresponding author: Sophie Faye, Abt Associates Inc, 4550 Montgomery Avenue Suite 800, Bethesda, MD, USA; Tel: 0013013475009; E-mail: sophie faye@abtassoc.com

Received October 13, 2017; Accepted October 19, 2017; Published October 26 2017

Citation: Faye S, Johns B, Baruwa E (2017) Costs and Efficiency of Integrating Family Planning into Antiretroviral Therapy Services in Zambia: A Mixed Method Comparison of the Fully Integrated Versus Referral Models. J AIDS Clin Res 8: 738. doi: $10.4172 / 2155-6113.1000738$

Copyright: (C) 2017 Faye S, et al. This is an open-access article distributed unde the terms of the Creative Commons Attribution License, which permits unrestricted use, distribution, and reproduction in any medium, provided the original author and source are credited. 


\section{Methods}

\section{Study design and sample selection}

This study is a cross-sectional, non-randomized comparison designed to test the cost-efficiency of the OSS and IR integration models. The sample of 10 health facilities was chosen from the districts where the Zambia Prevention, Care and Treatment Partnership II (ZPTC II) project and the Center for Infectious Disease Research in Zambia (CIDRZ) were supporting FP/HIV integration. The sample included six ZPTC II facilities (three implementing the OSS model and three implementing the IR model) and four supported by CIDRZ (all four implementing the IR model).

The integration models are implemented at health center level, which typically has several clinics including an HIV clinic and an FP clinic. In both integration models, FP counselling is systematically offered to female ART patients of reproductive age on ART care regardless of their FP status. Under the OSS model, if a method need is identified following the counselling, it is offered directly from the HIV clinic while under the IR model patients are referred to the FP clinic. Our analysis is limited to short term methods (pills, condoms, injectable) because they were the only methods available under both the OSS and IR models. The specific service delivery points this study considers for costing purpose are the HIV clinic and the FP clinic.

\section{Study measures and data collection}

This study assessed cost-efficiency of the different models through the unit cost per ART patient provided with FP services. All things being equal, a lower unit cost corresponds to increased cost-efficiency. From the FP/ART integration design, all women of reproductive age visiting the HIV clinic for ART care should receive FP counselling. If such service is not provided, this can lead to missed opportunities and a lower volume of integrated services (output); hence a decrease in costefficiency. We also estimated and compared that percentage of missed opportunities for FP counselling across models to assess the level of implementation for each integration model.

As part of this study, we used several data collection methods:

Patient exit interview: Based on an interview time of $25 \mathrm{~min}$ per patient and a one-day data collection period per health facility, 15 women aged 18-49 years were interviewed from each HIV clinic (150 interviews in total). They were randomly selected on the day of data collection. The questionnaire covered patient characteristics (age, education level and FP status) and FP service-related topics received during the visit.

Medical record review: At each ART clinic, a random sample of 90 patient records was selected (900 records in total). We calculated the sample size based on a confidence level of 95 percent, a desired margin of error of 10 percent and a proportion of ART patients counseled on FP of 38 percent [13]. To be part of the sample, patients had to be women 18-49 years of age who were actively on ART care, and who had visited the HIV clinic at least once since the start of the FP integration program.

Semi-structured providers interview: At each facility, the survey team interviewed the responsible officer-in-charge of the HIV clinic and a health worker directly interacting with patients as part of ART care (20 interviews in total). The team used a standardized guide to lead points of discussion. Information was collected about the current integration implementation (how it works, what training was offered, etc.), the enabling factors of integration, and the potential barriers to effective integration.
Cost data: The team collected cost and utilization data for a period of 12 months, from October 2013 to September 2014. The team also collected health facility characteristics (location, ownership, partners, etc.) and information on the level and type of inputs and outputs. Data on the volume of services offered by type was collected from the Health Monitoring Information System (HMIS). The team obtained the quantities of input (labor and drugs and supplies) from the facility/ clinic records and their unit price from the District Medical Office. The team also reached out to ZPTC II and CIDRZ to obtain costs related to training and supervision for the integration programs.

\section{Analytic approach}

We estimated the integration costs from the provider perspective using a combination of step-down and bottom- up approaches [14]. For the calculation of missed opportunities, we planned to estimate and compare data from the patient record review and from the patient exit interview. However, in the HIV clinics, data on FP services provision was inconsistently recorded in patient files, so we estimated the percentage of missed opportunities from the patient exit interview only. The percentage of missed opportunities for FP counselling was calculated as the proportion of female patients of reproductive age who did not get FP counselling in the HIV clinic out of those not currently using an FP method and expressing a need for FP. Need for FP, adapted from the standard Demographic and Health Survey definition [15], was described as a married woman with a partner or sexually active in the last three months, not pregnant and who did not want a child in the next two years. The information on FP needs was obtained from the patient exit interview.

For the statistical analysis, we used a non-parametric Mann Whitney $U$ test to compare unit costs of FP services provision and percentage of missed opportunities across integration models and across selected facility characteristics (district, implementing partner, number of clinical staff in HIV clinic, number of days the HIV clinic opens per week). We conducted the comparison across facility characteristics to see if there were other variables (different from the type of integration model) that could affect unit costs. The tests will be considered statistically significant if P-value is less than 5 percent. In addition to the tests, the team computed the relative difference in average unit costs for the two models which enables direct insight into the true scale of difference between the OSS and IR models. For qualitative providers' data analysis, the team used the interviewers' notes and transcripts from digital audio recording to identify the main constraints and enablers mentioned by providers in terms of frequency of occurrences.

This study received ethics approval from the Abt Associates Institutional Review Board and from the ERES CONVERGE Institutional Review Board in Lusaka, Zambia. Oral informed consent was obtained from patients as well as from facility managers and workers before any interviews took place.

\section{Results}

\section{Total and unit cost of services}

Focusing on the average share of drugs/supplies and labor across all facilities, we see a different cost structure between ART care and FP care in their respective stand-alone clinics.

For ART care, drugs and supplies constitute the majority of total costs $(94 \%)$ and labor represents only four percent of total costs. For FP care, we have the labor's share (73\%) dominating drugs and supplies 
Citation: Faye S, Johns B, Baruwa E (2017) Costs and Efficiency of Integrating Family Planning into Antiretroviral Therapy Services in Zambia: A Mixed Method Comparison of the Fully Integrated Versus Referral Models. J AIDS Clin Res 8: 738. doi: 10.4172/2155-6113.1000738

Page 3 of 5

(25\%). With such a low share of labor costs in the HIV clinic, the potential efficiency gains from adding FP to ART care (OSS model) should not be expected to be high because such efficiency gains would likely come from labor costs rather than from drugs and supplies costs. It should be noted that the level of these "efficiency gains" will depend on the magnitude of total costs.

In the OSS model, the average cost of ART care plus FP counselling per person per year was USD \$258 and USD \$261 for ART plus both counseling and FP method provision, slightly below the corresponding costs for the IR model (Table 1).

In general, the incremental cost of providing FP counselling in the ART clinic was low, as it did not necessitate many additional resources (mainly the provider training). Most of the cost difference resulted from the addition of FP method ( $\$ 7$ for the IR model and $\$ 3$ for the OSS model). The relative difference in average unit costs showed that for a given unit cost of FP method provision in the OSS model, the corresponding cost for the IR model was 2.3 percent more. The mean comparison test across the two models had a p-value of 0.57 for unit cost of FP counselling and a p-value of 0.36 for both FP counselling and method provision. Thus, neither model was found to be more costefficient than the other.

The results for unit cost of FP services were also presented

\begin{tabular}{|c|c|c|}
\hline & \multicolumn{2}{|c|}{ Average annual unit cost per patient } \\
\hline & $\begin{array}{c}\text { ART+FP } \\
\text { counselling (USD) }\end{array}$ & $\begin{array}{l}\text { ART+FP counselling+FP } \\
\text { method (USD) }\end{array}$ \\
\hline IR model & 260 & 267 \\
\hline OSS model & 258 & 261 \\
\hline$P$ values & 0.57 & 0.36 \\
\hline Difference in averages & $\$ 2$ & $\$ 6$ \\
\hline $\begin{array}{c}\text { Relative Difference in } \\
\text { averages }\end{array}$ & $0.8 \%$ & $2.3 \%$ \\
\hline
\end{tabular}

Table 1: Unit costs for FP services provision by integration model.

\begin{tabular}{|c|c|c|}
\hline & \multicolumn{2}{|c|}{ Average annual unit cost per patient } \\
\hline & $\begin{array}{c}\text { ART+FP } \\
\text { counselling (USD) }\end{array}$ & $\begin{array}{c}\text { ART+FP } \\
\text { counselling+FP } \\
\text { method (USD) }\end{array}$ \\
\hline \multicolumn{3}{|l|}{ Health facility District } \\
\hline Kabwe $(n=6)$ & 259 & 263 \\
\hline Mongu $(n=2)$ & 258 & 265 \\
\hline Lusaka $(n=2)$ & 260 & 268 \\
\hline p-value & 0.96 & 0.72 \\
\hline \multicolumn{3}{|l|}{ Implementing partners } \\
\hline $\operatorname{CIDRZ}(n=4)$ & 259 & 267 \\
\hline ZPTC II $(n=6)$ & 259 & 263 \\
\hline p-value & 0.78 & 0.93 \\
\hline \multicolumn{3}{|c|}{ Nbr. Clinical staff in HIV clinic } \\
\hline$<=2(n=5)$ & 262 & 267 \\
\hline$>2$ and $<=4(n=3)$ & 257 & 263 \\
\hline$>4(n=2)$ & 253 & 259 \\
\hline$p$-value & 0.12 & 0.10 \\
\hline \multicolumn{3}{|c|}{$\begin{array}{l}\text { Nbr. Days HIV clinic opens per } \\
\text { week }\end{array}$} \\
\hline $1(n=2)$ & 263 & 269 \\
\hline $2(n=5)$ & 258 & 262 \\
\hline $3(n=3)$ & 258 & 265 \\
\hline p-value & 0.45 & 0.44 \\
\hline
\end{tabular}

Table 2: Unit costs for FP services and facility characteristics. across other characteristics of the health facilities (Table 2). None of the variables significantly influenced unit costs: district $(\mathrm{P}>=0.72$, implementing partner $(\mathrm{P}>=0.78$, clinical staff in HIV clinic $(\mathrm{P}>=0.1)$ and days HIV clinic opens per week $(\mathrm{P}>=0.4)$.

When assessing the unit costs against the volume of services, we did not see a clear link to the model implemented (Figure 1). HIV clinics with higher patient volume have lower unit cost regardless of model.

\section{Missed opportunities}

The average percentage of missed opportunities (Table 3 ) for the OSS model are lower than for the IR model (25\% versus $33 \%$ ), but the difference were not statistically significant $(\mathrm{P}$ value $=0.3$ ).

These averages also have wide confidence intervals, indicating wide variation across clinics. These observed level of missed opportunities raise the question of implementation according to design for the integration models included in this study.

The relative difference value is more than triple the absolute difference suggesting that for example, for every missed opportunities occurring in the OSS model, 32 percent more missed opportunities occur in the IR model.

\section{Barriers and enablers to integration}

Analysis of the qualitative provider interviews found that "shortage of staff" is a key recurrent concern with integration. All of the health workers and managers interviewed noted that clinical providers were overworked, and that more clinical staff is needed to successfully integrate FP into ART care. Some facility in-charge officers also noted that the importance of providing FP counseling in addition to ART care was not always well understood by the clinical staff: "The integration

\begin{tabular}{|l|c|c|}
\hline \multirow{2}{*}{} & $\begin{array}{c}\% \text { missed opportunities for non-FP users } \\
\text { with identified FP needs }\end{array}$ \\
\cline { 2 - 3 } & Average & $\begin{array}{c}\text { 95\% Confidence } \\
\text { interval }\end{array}$ \\
\hline IR model & $33 \%$ & \multicolumn{2}{|c|}{$13 \%-53 \%$} \\
\hline OSS model & $25 \%$ & \multicolumn{2}{|c|}{$0.39 \%$} \\
\hline P values & \multicolumn{2}{|c|}{$8 \%$} \\
\hline Absolute Difference in averages & \multicolumn{2}{|c|}{$32 \%$} \\
\hline Relative Difference in averages & \multicolumn{2}{|c|}{$3 \%$} \\
\hline
\end{tabular}

Note: Confidence intervals were calculated using robust standard errors Data source: Patient exit interview

Table 3: Percentage of missed opportunities for FP counseling.

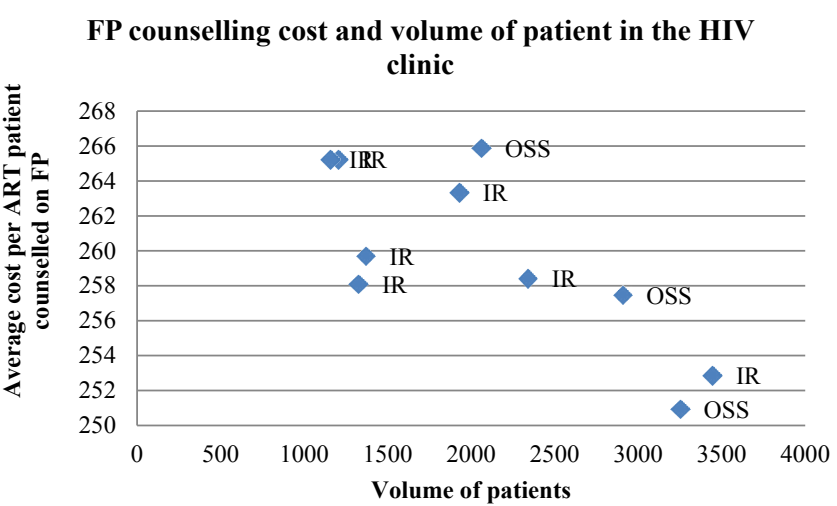

Figure 1: Unit costs for FP counselling and HIV clinic patient volume. 
intervention is not quite a success, because sometimes the clinical staff fails to give the FP information to the clients. It is as if they put more importance on the ART care. If you look at the patient record you can see that."

Infrastructure constraints, such as inadequate space for privacy in the ART clinic, were another barrier that the providers mentioned, mainly for the OSS model. Providers implementing the OSS model were also trained to provide long-term methods, but they noted that integration of those services had not yet started, due to the lack of a private space in the ART clinic.

The lack of a formal referral system was seen as a challenge in the integration process mainly for the IR model. Health workers noted the difficulties in trying to track the patients they refer to the FP clinic, because there was no formal referral tracking or counter-referral system included in the integration design.

In terms of enablers, seven out of ten facility in-charges identified the provision of enough up-front information about the integration process as one of the necessary elements for success. Providers generally appreciated the new skills they acquired through training and stressed the importance of this knowledge in caring for their ART patients. Eighty five percent of interviewed providers appreciated the fact that the implementing partners (ZPTC II and CIDRZ) made regular supervision visits.

\section{Discussion}

This study set out to estimate costs and compare cost-efficiency of two models of integrating FP and HIV services: the fully integrated OSS model and the internal referral model and using data collected in 10 facilities in Zambia, we concluded that there was no significant difference in cost-efficiency between these two models.

In the Zambian setting, providing FP service delivery in addition to ART care had a relatively low financial cost that mainly includes training and supervision. Under both integration models, the FP clinic in a given health facility will still need to function to serve the HIV negative population needing FP services. Thus, from a provider's perspective, unless the population of ART patients in need of FP services and treated in the HIV clinic is very large, we are unlikely to see significant cost-efficiency gains from fully integrating ART and FP services. This finding of modest potential efficiency gains was also reported by Obure et al. [10] who concluded in their study that "contrary to expectation, efficiency gains from the integration of HIV and SRH services, if any, are likely to be modest" (p. 132). Moreover, most of the efficiency gains from integration are likely to come from labor costs, which get distributed among a higher volume of services, and in our study we have seen that labor costs constitute a low share of total costs for the HIV clinics.

One possible explanation for our finding of no difference in costefficiency among the two models might be the extent to which under the integration models, patients are systematically given FP services or not. Missed opportunities for FP services, especially if the patient has unmet FP needs, can considerably decrease the output of the integration models, hence their cost-efficiency. This situation could arise for different reasons, but the one more frequently noted by providers was the larger focus of the HIV clinic staff on ART care (often to meet aggressive targets for care provision) . Regardless of the causes, missed opportunities for FP services for these FP/ART integration models should be addressed for the investment made to generate the desired outcomes.
Another implementation challenge is the lack of consistent recording of the FP services provided as we found from the patient record review. Providers could be offering the services, but if they do not record them properly it will be difficult to track and measure the volume. This lack of consistent data prevents rigorous impact analysis and diminishes implementing partners' ability to scale up the programs based on evidence. For the IR model, consistent recording of services received in the HIV clinic is not even enough. Without a formal referral system to track the patient from the HIV to the FP clinic, it will be difficult to measure the impact of the IR model on FP method uptake. Such a formal referral system was shown to have great success under the ZPTC II project. Between September and November 2013, ZPCT II piloted a new system of tracking referrals from HIV to FP services in 15 clinics, focusing on quantifying the number of clients referred and tracking the uptake of FP from such referrals. The results concluded that with an enhanced referral and tracking system, it is feasible to demonstrate very good uptake of FP services ( $85 \%$ of those referred) by clients accessing HIV services where fully integrated HIV/FP services are not available [16]. In a separate study in Tanzania, Baumgartner et al. [17] evaluated a facilitated referral system between the HIV and FP clinics where, in addition to the formal referral system, their model included physically accompanying the patient from the HIV to the FP clinic. The authors found that the proportion of sexually active clients using a contraceptive method post-intervention increased by an estimated 12 percent $(\mathrm{P}=0.013)$ compared to before the intervention.

\section{Limitations}

Some limitations of this analysis need to be noted. First, we do not account for quality in our analysis so the two models are assumed to be providing similar quality of service. Second, because of a lack of disaggregated data, the cost per patient receiving an FP method was calculated as an average for all short-term contraceptive methods instead of for each type of method. Third, because of our modest sample size, the results obtained from this study lack the statistical power of larger datasets which limits the representability of the results. Finally, as with most studies of this kind, we cannot establish strong causality due to the use of non-experimental design, which can suffer from limitations such as selection bias inherent to the weaker design. However, the approach we adopted is commonly accepted as a sound basis for exploring associations [10].

\section{Conclusion}

Despite these limitations, our findings have policy implications for the planning and implementation of FP/ART integration programs. The results of this study show that fully integrated model of FP/ART services is not necessarily more cost-efficient than the referral model. The results also indicate that the performance of each can depend on particular settings such as the volume of ART patients to be served, the level of missed opportunities, and the strength of the referral system. Moreover, the results indicate that there is still room to improve efficiency of these current models in Zambia through a decrease in missed opportunities for FP services.

Overall, the results of this study emphasize the need for more research to understand how to improve the implementation of integration models and increase cost-efficiency of FP/HIV integration. There is a need for larger controlled experiment type studies to better understand the outcome of different ways of ensuring that HIV positive women can get the reproductive health care they need in countries with high HIV prevalence like Zambia. 
Citation: Faye S, Johns B, Baruwa E (2017) Costs and Efficiency of Integrating Family Planning into Antiretroviral Therapy Services in Zambia: A Mixed Method Comparison of the Fully Integrated Versus Referral Models. J AIDS Clin Res 8: 738. doi: 10.4172/2155-6113.1000738

Page 5 of 5

\section{Acknowledgement}

This article was prepared with support of the Daniel B McGillis Professional Development and Dissemination Award from Abt Associates. The work was funded by the USAID Office of HIVIAIDS and was executed by the Health Finance and Governance project. The authors are grateful to the people who made this study possible, including Ms. Kelley Ambrose, Dr. Peter Cowley, Dr. Laurel Hatt and Ms. Marianne El-Khoury from Abt Associates; Mr. Collen Zulu from the USAID mission in Zambia; Dr. Mwangelwa Mubiana and Mrs. Maureen Mzumara of the Center for Infectious Disease Research in Zambia; Dr. Prisca Kasonde of the Zambia Prevention, Care and Treatment Partnership II project and Mr. Petan Hamazakaza who led the data collection team in country.

\section{References}

1. WHO (2009) Strategic considerations for strengthening the linkages between family planning and HIVIAIDS policies, programs and services. World Health Organization, USAID and Family Health International

2. MacQuarrie KL (2015) HIVIAIDS and unmet need for family planning. DHS Working Papers, p: 122.

3. Halperin DT, Stover J, Reynolds HW (2009) Benefits and costs of expanding access to family planning programs to women living with HIV. AIDS 23: S123-S130.

4. Calvert C, Ronsmans C (2013) The contribution of HIV to pregnancy-related mortality: A systematic review and meta-analysis. AIDS 27: 1631-1639.

5. Manuela CM, Mutemwa R, Kivunaga J, Stackpool Moore L, Mayhew SH, et al. (2014) Experiences of stigma among women living with HIV attending sexual and reproductive health services in Kenya: A qualitative study. BMC Health Serv Res 14: 412.

6. Wilcher R, Hoke T, Susan E, et al. (2013) Integration of family planning into HIV services: A synthesis of recent evidence. AIDS 27: S65-S75.

7. Lindegren ML, Kennedy CE, Bain-Brickley D, Azman H, Creanga AA, et al. (2012) Integration of HIVIAIDS services with maternal, neonatal and child health, nutrition and family planning services. Cochrane Database Sys Rev 12: 9.
8. Kennedy CE, Spaulding AB, Brickley DB, Almers L, Mirjahangir J, et al. (2010) Linking sexual and reproductive health and HIV interventions: A systematic review. J Int AIDS Soc 13: 26.

9. Church K, Mayhew SH (2009) Integration of STI and HIV prevention, care, and treatment into family planning services: A review of the literature. Stud Fam Plann 40: 171-186.

10. Obure CD, Guinness L, Sweeney S, et al. (2016) Does integration of HIV and SRH services achieve economies of scale and scope in practice? A cost function analysis of the Integra Initiative. Sex Transm Infect 92: 130-134

11. Sweeney S, Obure CD, Maier CB, Greener R, Dehne K, et al. (2012) Costs and efficiency of integrating HIVIAIDS services with other health services: A systematic review of evidence and experience. Sex Transm Infect 88: 85-99.

12. Shade SB, Kevany S, Onono M, Ochieng G, Steinfeld RL et al. (2013) Cost cost-efficiency and cost-effectiveness of integrated family planning and HIV services. AIDS 27: S87-S92.

13. Adamchak S, Janowitz G, Liku J, Munyambanza E, Grey T, et al. (2010) Study of Family Planning and HIV integrated services in five countries: Final report. Family Health International Research Triangle Park, NC.

14. Drummond M, Sculpher M, Claxton K, Greg L, Torrance G (2005) Methods for the economic evaluation of healthcare programmes. Oxford University Press Oxford.

15. Bradley S, Croft T, Fishel J, Charles F (2012) Revising unmet need for family planning. ICF International, Calverton, MD.

16. Kasonde P, Koni P, Malebe T, Dulli L, Chishinga N, et al. (2014) Enhanced systems for tracking referrals to FP from HIV services - Does It Help Increase Uptake of FP Services? XX International AIDS Conference; Melbourne.

17. Baumgartner JN, Green M, Weaver MA, Mpangile G, Kohi TW, et al. (2014) Integrating family planning services into HIV care and treatment clinics in Tanzania: Evaluation of a facilitated referral model. Health Policy Plan 29: 570579. 